\title{
The CaMKII/NMDAR complex as a molecular memory
}

Magdalena Sanhueza ${ }^{1}$ and John Lisman ${ }^{2^{*}}$

\begin{abstract}
CaMKII is a major synaptic protein that is activated during the induction of long-term potentiation (LTP) by the $\mathrm{Ca}^{2+}$ influx through NMDARs. This activation is required for LTP induction, but the role of the kinase in the maintenance of LTP is less clear. Elucidating the mechanisms of maintenance may provide insights into the molecular processes that underlie the stability of stored memories. In this brief review, we will outline the criteria for evaluating an LTP maintenance mechanism. The specific hypothesis evaluated is that LTP is maintained by the complex of activated CaMKII with the NMDAR. The evidence in support of this hypothesis is substantial, but further experiments are required, notably to determine the time course and persistence of complex after LTP induction. Additional work is also required to elucidate how the CaMKII/NMDAR complex produces the structural growth of the synapse that underlies late LTP. It has been proposed by Frey and Morris that late LTP involves the setting of a molecular tag during LTP induction, which subsequently allows the activated synapse to capture the proteins responsible for late LTP. However, the molecular processes by which this leads to the structural growth that underlies late LTP are completely unclear. Based on known binding reactions, we suggest the first molecularly specific version of tag/ capture hypothesis: that the CaMKII/NMDAR complex, once formed, serves as a tag, which then leads to a binding cascade involving densin, delta-catenin, and $\mathrm{N}$-cadherin (some of which are newly synthesized). Delta-catenin binds AMPA-binding protein (ABP), leading to the LTP-induced increase in AMPA channel content. The addition of postsynaptic $\mathrm{N}$-cadherin, and the complementary increase on the presynaptic side, leads to a trans-synaptically coordinated increase in synapse size (and more release sites). It is suggested that synaptic strength is stored stably through the combined actions of the CaMKII/NMDAR complex and N-cadherin dimers. These N-cadherin pairs have redundant storage that could provide informational stability in a manner analogous to the base-pairing in DNA.
\end{abstract}

CaMKII is a highly abundant brain protein concentrated in the postsynaptic density (PSD) and is strongly implicated in LTP (reviewed in [1]). During the induction of LTP, $\mathrm{Ca}^{2+}$ enters through the NMDAR and binds to calmodulin [2]. Calmodulin then activates CaMKII, which phosphorylates the GluA1 subunits of AMPARs and an auxiliary subunit of AMPARs, stargazin. The first reaction increases the conductance of AMPARs [3,4]; the second allows more AMPARs to be bound into the synapse by PSD-95 [5,6]. Together, these processes provide a mechanistic explanation for the early phase of LTP (approximately the first 30-60 minutes). Later phases of LTP appear to require different mechanisms, and it is these mechanisms that maintain LTP for the long periods

\footnotetext{
* Correspondence: Lisman@brandeis.edu

${ }^{2}$ Volen Center for Complex Systems, Biology Department, Brandeis University, 415 South Street, MS 008, Waltham, MA 02454-9110, USA

Full list of author information is available at the end of the article
}

required for memory storage. The hypothesis that we will evaluate in this review is that late LTP is maintained by the complex of CaMKII with the NMDAR. Evidence relevant to the following criteria will be summarized:

1. LTP induction should cause a persistent increase in the CaMKII/NMDAR complex.

2. Inhibiting formation of the CaMKII/NMDAR complex should block LTP induction.

3. Decreasing the amount of CaMKII/NMDAR complex after LTP induction should reverse LTP.

4. A component of basal transmission should be reversed by reducing the basal CaMKII/NMDAR complex.

5. There must be a mechanism by which CaMKII/ NMDAR complex can produce potentiation, specifically the trans-synaptic structural processes that underlie late LTP. 


\section{LTP induction should cause a persistent increase in the CaMKII/NMDAR complex}

The initial evidence that CaMKII could interact with the NMDAR came from in vitro experiments showing that a fragment of GluN2B is a substrate for purified CaMKII (at 1303) [7]. It was noted that the binding affinity of GluN2B was much higher than for other CaMKII substrates. Subsequent experiments showed that CaMKII could form a tight complex with NMDAR and that the complex was present in living cells, as identified by crosslinking or coimmunoprecipitation (co-IP) [8-10]. Importantly, the amount of complex was increased substantially by stimulation that elevated intracellular $\mathrm{Ca}^{2+}$ and thereby activated CaMKII.

A critical assumption of the proposed model is that LTP produces an increase in the CaMKII/NMDAR complex that is persistent for the duration of LTP. This assumption remains to be tested directly, but there is relevant evidence. Stimulation of cultured neurons with glutamate/glycine (a form of chemical LTP) can trigger persistent translocation of CaMKII that requires kinase binding to GluN2B at what is termed the T-site [11]. Importantly, the persistently translocated CaMKII is phosphorylated at T286 [12]. Furthermore, chemical LTP produces translocation of CaMKII to the PSD, as visualized by electron microscopy; this persists for at least $1 \mathrm{hr}$ after LTP induction, suggesting that there is persistent formation of the CaMKII/NMDAR complex [13,14].

The binding of CaMKII to the NMDAR requires an activated open form of the kinase. Therefore, the recent optical experiments [15] demonstrating that CaMKII is only transiently activated after LTP induction $(\sim 1$ minute) could suggest that the CaMKII/ NMDAR complex is also transient. However, there are two reasons to doubt the generality of this conclusion. First, recent measurements indicate that the fraction of CaMKII subunits directly bound to the NMDAR and thereby locked in the open confirmation [16] is a small fraction of the total CaMKII in spines and may be difficult to detect optically [17]. Second, the optical measurements of CaMKII activation were made under conditions that did not evoke late LTP [18], raising the possibility that future measurement under conditions that did induce late LTP would evoke a detectable persistent component, as seen with induction protocols based on tetanic stimulation [19]. In any case, given that the key question is the duration of the LTP-induced increase in CaMKII/NMDAR complex, what is needed is a method (possibly based on FRET) that would make it possible to directly monitor the kinetics of complex formation and persistence.

\section{Inhibiting formation of the CaMKII/NMDAR complex should block LTP induction}

To examine the functional role of complex formation, Barria and Malinow [20] overexpressed a form of GluN2B having mutations near the CaMKII phosphorylation site (R1300Q and S1303D) that strongly interferes with binding [21]. LTP was examined using a low-frequency pairing protocol. There was a significant (but not complete) block of potentiation in the first 10 minutes after LTP induction; at later times, the block was complete.

Related experiments were conducted in hippocampal slices from knockin (KI) mice in which CaMKII-GluN2B binding was impaired by the mutations L1298A and R1300Q [22]. In these mice, the basal CaMKII/NMDAR complex was reduced by about $40 \%$. Activity-dependent formation of complex in cell cultures was more strongly reduced. LTP induced by high-frequency stimulation in slice experiments was reduced, but only by about $50 \%$. It is unclear whether the smaller reduction than in the Barria/Malinow experiments is due to a slightly different mutation, to the difference in animal age, or to a difference in the LTP induction protocol.

Another method for interfering with the CaMKII/ NMDAR complex utilized transgenic mice in which the whole C-terminal GluN2B fragment could be induced [23]. The LTP induced by high-frequency stimulation was reduced by about $50 \%$. It is unclear whether this reduction was due to inhibiting CaMKII activity or to inhibiting the formation of the CaMKII/NMDAR complex.

A complementary experiment would be to determine the effects of increasing the CaMKII/NMDAR complex. Overexpression of activated CaMKII holoenzyme increases synaptic strength and spine size, provided that phosphorylation of T305/306 is prevented [24]. It would be expected that this form of holoenzyme would increase the CaMKII/ NMDAR complex and synapse size, but this has not been directly tested.

\section{Decreasing the amount of CaMKII/NMDAR complex after LTP induction should reverse LTP}

An important tool has been the development of a related group of peptides (CN27; CN21; CN19) that block the formation of the CaMKII/NMDAR complex in vitro [25]. This peptide family derives from a fragment of an endogenous CaMKII inhibitor protein, CaMKIIN [26]. This protein strongly binds to a site on CaMKII (the T-site) in addition to the catalytic site to which other peptide inhibitors mainly bind (these are based on the sequence of the CaMKII regulatory domain). It is to the $\mathrm{T}$-site that the NMDAR itself binds.

Application of CN21 can reverse LTP in hippocampal slices [27], as shown in Figure 1. An action of this kind would be consistent with an effect on either LTP expression or maintenance processes. An experiment that 


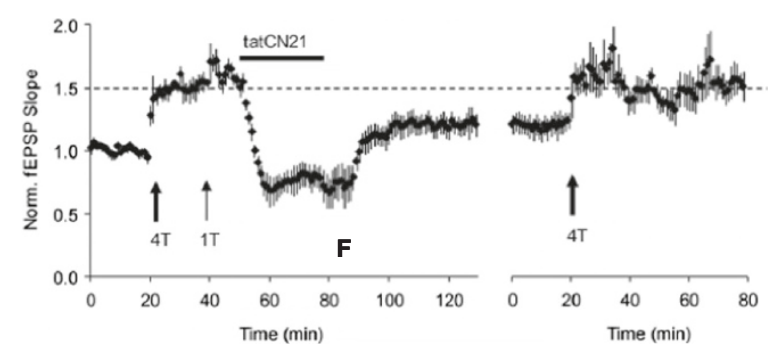

Figure 1 TatCN21 reverses LTP. fEPSPs recorded from the CA1 region of a hippocampal slice. At $20 \mathrm{~min}$, LTP was induced by four tetani. This LTP was saturated, as evidenced by lack of further potentiation when an additional tetanus was given at $40 \mathrm{~min}$. tatCN21 was then applied for $30 \mathrm{~min}$. Upon removal, there was partial recovery, but also a non-recoverable component that demonstrates LTP reversal. To verify that saturated LTP had indeed been reversed, an additional tetanus was given (right), and this reinduced LTP. In control experiments without tatCN21 application, LTP could not be reinduced.

distinguishes between these possibilities is to remove the peptide; if the effect was on expression, LTP should recover. Figure 1 shows that LTP does not return. It could be argued that this lack of return resulted from damage; however, the ability to then reinduce LTP argues against this possibility. Thus, CN21 appears to reset a molecular switch that controls LTP maintenance.

Several controls point to the fact that CN21 works in slices to reduce the CaMKII/NMDAR complex, as expected from in vitro work. First, the peptide reduced the amount of basal complex, as measured by co-IP (basal transmission was also reduced; see next section). If somewhat lower concentrations of CN21 were used $(5 \mu \mathrm{M}$ instead of $20 \mu \mathrm{M})$, there was no reduction in the CaMKII/NMDAR complex and no persistent reduction in basal transmission. Second, the peptide produced a small but statistically significant reduction in the amount of CaMKII bound in spines. Third, CN21 produced a large reduction in the activity-dependent increase of CaMKII in the PSD [28].

The ability of CN compounds to rapidly reverse LTP is not shared by other peptides that primarily inhibit the CaMKII catalytic site [29,30]. This suggests that the reversal is due to interference with structural rather than enzymatic reactions. This could mean that there is no role for enzymatic activity (including autophosphorylation-induced autonomous activity [16]) during LTP maintenance. Alternatively, the effects of inhibiting such activity may require many hours to develop and thus have not been seen in the much shorter experiments performed thus far. Indeed, experiments have shown that although phosphatase is present in the PSD, there is negligible dephosphorylation of CaMKII T286 within $1 \mathrm{hr}$ [31].

\section{A component of basal transmission should be reversed by reducing the basal CaMKII/NMDAR complex}

LTP has been observed in vivo during learning [32,33]. Moreover, synapses that have undergone learningdependent LTP cannot then undergo LTP by electrical stimulation. This occlusion suggests that learning has saturated the normal LTP process. It would thus be expected that at least part of basal transmission, as measured in hippocampal slices, is the result of LTP-like events that occurred while the animal was alive. This inference is confirmed by analysis of unitary responses in the slice: the stronger the basal transmission, the smaller the magnitude of the LTP that can be induced [34,35].

Based on the data outline in the above paragraph, a strong expectation is that an agent that affects a synaptic memory mechanism should reduce basal transmission. Consistent with this, $\mathrm{CN}$ compounds depress basal transmission [27]. Moreover, this depression persists after removal of the $\mathrm{CN}$ compound, and the LTP that can then be induced is larger than if a scrambled control peptide or no peptide had been applied.

$\mathrm{CN}$-induced depression is observed for drug concentrations necessary to disrupt basal CaMKII-NMDAR interaction in the slices, but not for lower concentrations causing only kinase inhibition [27]. Moreover, this type of depression is different from known forms of activitydependent long-term depression (LTD), and it does not require $\mathrm{Ca}^{2+}$ influx, protein synthesis, or degradation [36]. The CN-induced persistent depression was barely detected in very young animals (P7-P10; [36]) for which CaMKII content at synapses is comparatively much lower $[37,38]$. Taken together, these findings strongly suggest that $\mathrm{CN}$-induced depression of basal transmission is caused by breakdown of the CaMKII-NMDAR interaction at the synapse. Perhaps inconsistent with this conclusion is the fact that basal transmission was not reduced when the basal level of CaMKII/NMDAR complex was reduced by knockin of NMDAR mutations [22]. However, it is difficult to interpret such knockin experiments because known homeostatic processes could have normalized transmission over the lifetime of the animal.

\section{There must be a mechanism by which CaMKII/ NMDAR complex can lead to the trans-synaptic structural growth that underlies late LTP}

LTP has an early phase that does not involve synapse growth and does not require protein synthesis; this is followed within $\sim 1 \mathrm{hr}$ by late LTP, which involves synapse growth and requires protein synthesis [39,40]. An important concept is the tag and capture model [41]. According to this model, strong stimulation leads to "tagging" of the stimulated synapse by addition of a 
protein; this then serves to capture newly synthesized proteins, leading to late LTP at the activated synapse. Evidence suggests that CaMKII is the tag $[42,43]$.

Relatively little is known about how proteins produce the synaptic growth that underlies late LTP. One area of progress has established a key role for $\mathrm{N}$-cadherin, a homophilic adhesion molecule that forms a trans-synaptic linkage between the presynaptic and postsynaptic sides of the synapse. Pharmacological experiments, gene knockout experiments, conditional knockout experiments, and RNAi experiments all show that $\mathrm{N}$-cadherin is required for late LTP (but not for early LTP) [44-46]. Furthermore, LTP induction causes synthesis of $\mathrm{N}$-cadherin and its insertion into the synapse [44]. Finally, electron microscopy shows that overexpression of $\mathrm{N}$-cadherin can increase synapse size [47]. Thus, although there are many forms of adhesion molecules at synapses, $\mathrm{N}$-cadherin appears to be of central importance in late LTP.

There have been no previous suggestions about the cascade of biochemical events by which LTP induction results in the incorporation of $\mathrm{N}$-cadherin into the synapse. Figure 2 shows a working hypothesis based on known binding interactions. It is proposed that the CaMKII/NMDAR complex acts as a structural seed for a series of binding reactions that gradually increase synapse size and strength, thereby accounting for late LTP. The following known binding interactions could be involved: densin-180 (and actinin) binds to the CaMKII in the CaMKII/NMDAR complex [48,49]; delta-catenin binds to densin [50]; and N-cadherin binds to delta-catenin [51]. As noted above, overexpression of N-cadherin leads to synapse growth. We thus posit that the addition of Ncadherin to the synapse during late LTP similarly leads to synapse growth. An important constraint on any model of synapse growth is that it must be trans-synaptically coordinated, leading to precise registration of the edges of the presynaptic grid and postsynaptic density [52]. The role of the N-cadherin trans-synaptic dimers in organizing synaptic growth provides a simple explanation of this coordination (Figure 3).

Physiological analysis suggests that late LTP involves both addition of AMPA receptors and an increase in presynaptic release sites $[53,54]$. It is thus of interest that delta-catenin binds to AMPA-binding protein (ABP) [55] and may thereby increase the AMPA channel (notably GluA2) content of the synapse [56-58]. Ncadherin can also bind directly to GluA2 [59], see also [60]. There is relatively little that can be said about the presynaptic changes that lead to enhanced vesicle release. It is known that overexpression of postsynaptic N-cadherin affects presynaptic function [61], but how this happens is unclear. Perhaps presynaptic N-cadherin is brought into the synapse simply by binding to postsynaptic N-cadherin. Alternatively, insertion of presynaptic $\mathrm{N}$-cadherin might depend on the presynaptic activation of CaMKII [62], translocation of CaMKII to the pre-

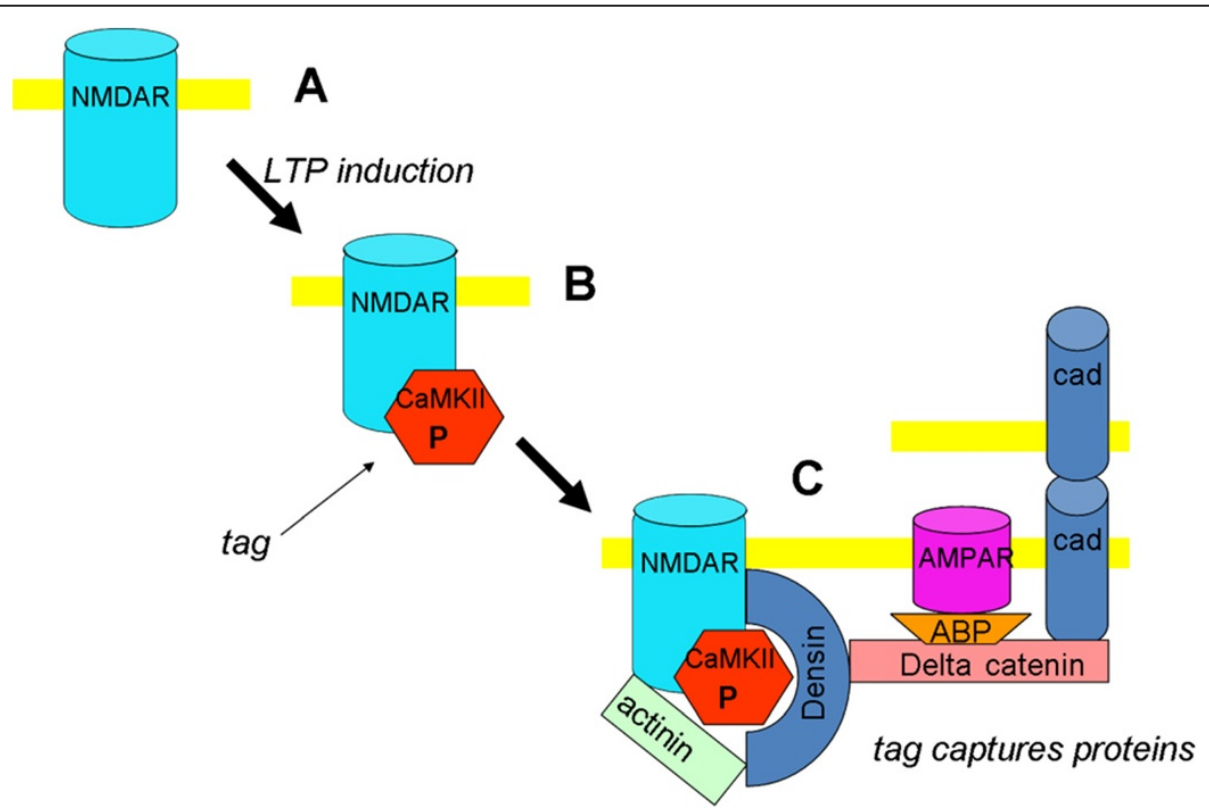

Figure 2 Working hypothesis for how formation of CaMKII/NMDAR complex during LTP induction leads to subsequent binding reactions necessary for late LTP. A, Before LTP induction (some NMDA channels have no CaMKIl bound). B, During LTP induction, CaMKII is activated and forms a persistent complex with the NMDA channel, thus forming a tag. $\mathbf{C}$, This serves as a structural seed for the gradual capture of densin, delta-catenin, ABP, and N-cadherin (cad). The addition of ABP provides additional anchoring sites for AMPA channels, thereby strengthening transmission. The binding of additional $\mathrm{N}$-cadherin (which is synthesized in response to activity) enlarges the synapse both presynaptically and postsynaptically. 


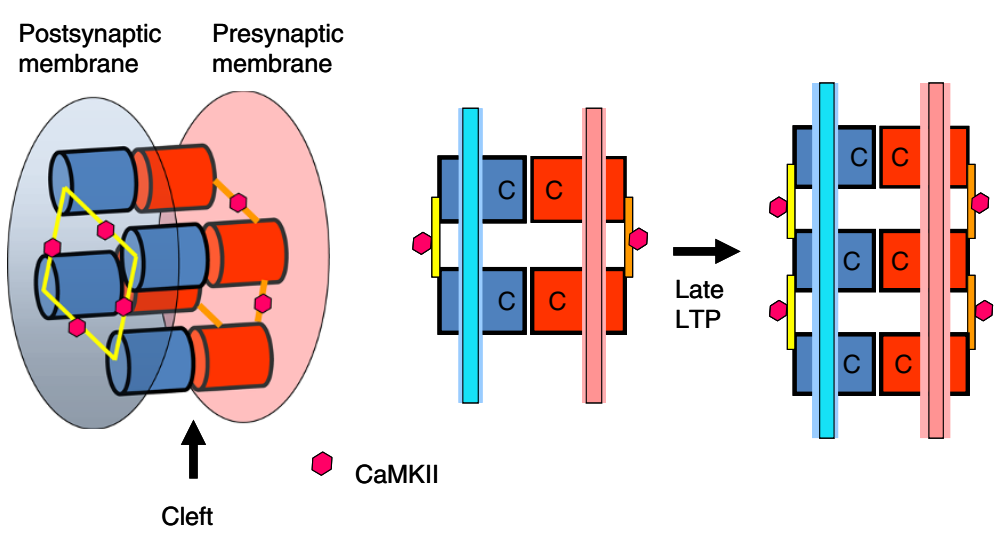

Figure 3 Model of trans-synaptic growth. Presynaptic (red) and postsynaptic (blue) cadherins (C) form homophilic bonds in the synaptic cleft (analogous to base-pairing in DNA). Cadherins are crosslinked by proteins in the postsynaptic density and presynaptic grid (yellow, orange), including CaMKII (red hexagons), which are analogous to the backbone of the DNA strands. Middle 2-D picture emphasizes analogy to DNA. Synapse size and strength are determined by the number of $\mathrm{N}$-cadherin dimers. This number is redundantly stored by presynaptic and postsynaptic cadherin arrays and increases during late LTP.

synaptic grid [63], and a protein linkage to $\mathrm{N}$-cadherin [51] analogous to the postsynaptic linkage described in Figure 2.

It is noteworthy that Figure 3 suggests a limited analogy to DNA: just as in DNA, there are two crosslinked backbone structures attached to elements that link the two backbone structures by a pairing rule (in DNA, the rule is heterologous; here, it is homologous). The existence of two backbone structures provides redundant information storage. Thus the N-cadherin dimers could contribute to informational stability, just as base-pairing does in DNA. Specifically, if one member of an Ncadherin dimer was lost in the process of turnover, the existence of the other member could serve as a basis for repair. In this way, the number of $\mathrm{N}$-cadherin dimers could be stable despite turnover of N-cadherin. This stability, in turn, could help to stabilize the CaMKII/ NMDAR complex, as described in the next paragraph.

Although we have posited that the CaMKII/NMDAR complex brings about the capture of the protein complex that includes actinin, densin, delta-catenin, and $\mathrm{N}$ cadherin, the complex, once established, may be important for the stabilization of CaMKII/NMDAR complex during protein turnover. During turnover of CaMKII, phosphorylated CaMKII holoenzymes may be removed from the NMDAR, leaving a pocket formed by densin and actinin. This pocket is relatively stable because it is held there by other parts of the complex that include the $\mathrm{N}$-cadherin dimmers (Figure 2). The phospho-CaMKII that left the pocket can be replaced by CaMKII that is either not phosphorylated (but with $\mathrm{Ca}^{2+} /$ calmodulin bound) or weakly phosphorylated. There, CaMKII can bind to GluN2B [11] and will become autophosphorylated as a result of stimulation by actinin [64] and GluN2B [16] and because PSD CaMKII is protected from phosphatase
[31] (probably because of the protein structure around bound CaMKII). This highly phosphorylated state then leads to tighter binding to the NMDAR [11]. In this way, the original tight complex of CaMKII bound to the NMDAR can be restored despite the molecular turnover.

This model provides an explanation for why overexpression of catalytically dead CaMKII can reverse memory (see next section). Specifically, this form (K42M) can bind to the NMDAR (albeit weakly), but not undergo the autophosphorylation necessary for tight binding to the NMDAR [11] and densin [65]. Weak binding to the NMDAR and densin may ultimately lead to dissociation of the entire complex, resulting in loss of LTP and memory.

Our model leads to testable predictions: that densin180, delta-catenin, and N-cadherin are required for synapse growth and late LTP. As noted earlier, N-cadherin is required for late LTP (but not early LTP) and for synapse growth. Knockout of delta-catenin or densin does not block early LTP $[58,66]$; the effect on late LTP or synapse growth has not been tested.

\section{Effect of altering the CaMKII/NMDAR complex on behavioral tests of memory}

Evidence that CaMKII signaling contributes strongly to the maintenance of nonspatial forms of memory has recently been published. One line of work comes from research on addiction in rats [67]. It was found that a persistent reversal of drug-induced sensitization could be produced by transient expression of a kinase-dead CaMKII mutant (K42M). In these experiments, animals were initially subjected to repetitive amphetamine exposure. Subsequently, transient virally mediated expression of $\mathrm{K} 42 \mathrm{M}$ produced a persistent block of the normal longlasting behavioral response to the drug (enhanced loco- 
motion and drug self-administration). Because expression of $\mathrm{K} 42 \mathrm{M}$ had ceased at the time of testing, the action of K42M must have been to reset a memory switch. In addition to the lack of catalytic activity and autophosphorylation, K42M displays an altered pattern of synaptic translocation in cultured neurons. While transient translocation is not affected $[68,69]$, the strength of binding to the NMDAR is strongly decreased $[21,70]$. Thus, there is a reasonable basis for suspecting that $\mathrm{K} 42 \mathrm{M}$ can erase a memory by acting as a dominant negative for stable CaMKII/NMDAR complex. A further indication that CaMKII is involved in the maintenance of behavioral memory comes from the experiments of [71]. They found that conditioned fear could be erased by transient post-learning inhibition of overexpressed CaMKII. However, the inhibition was animal-wide, so the location and physiological correlate of this effect are unclear.

Recent experiments have sought to test directly the role of the CaMKII/NMDAR complex in memory. To address this issue, [22] utilized a knockin mouse with mutations L1298A and R1300Q in GluN2B that interfere with that interferes with the formation of the CaMKII/NMDAR complex. A key finding was that spatial memory in the Morris water maze task, when measured at 1 or 3 days after the last training session, was greatly reduced (memory 1-2 hr after each training session was not reduced). Interpretation of the results is complicated by the fact that the mutation only produced a partial reduction in the basal CaMKII/NMDAR complex and only a $\sim 50 \%$ reduction in LTP. It is thus possible that the learning that occurred in this task was due to this remaining ability of complex to form. It is also of interest to consider the possibility that CaMKII can form other complexes (i.e., not with NMDARs) that contribute to synaptic enhancement. Indeed, if presynaptic CaMKII is necessary for binding of $\mathrm{N}$-cadherin and growth of the presynaptic active zone, the binding of CaMKII to a presynaptic protein, possibly $\mathrm{Ca}^{2+}$ channels [72], could contribute to presynaptic structural changes during LTP. A further question is whether memory maintenance can be reversed by interfering with the CaMKII/NMDAR complex after learning. Only one study thus far deals with this issue [73]. It was found that a $\mathrm{CN}$ peptide injected into the cingulate cortex was able to reverse a form of central pain. Importantly, induction of central pain produced a large increase in the amount of CaMKII/NMDAR complex in PSDs of the cingulate, and this increase was reversed by $\mathrm{CN}$.

\section{The PKM-zeta alternative}

It is noteworthy that a major alternative hypothesis [74], that memory is stored by a persistent increase in PKM- zeta, has substantial weaknesses, many of which have only recently become apparent. A critical test of the effect of PKM-zeta to reverse LTP maintenance has not been done. While it was shown that LTP in the slice could be reduced by ZIP, it was not shown that this reduction persisted after removal of ZIP [75]. In different experiments in which ZIP was transiently injected in vivo, the drug was still present $2 \mathrm{hr}$ after injection, as shown by immunochemistry; no removal of drug was demonstrated [76]. Thus, the existing experiments do not distinguish between effects on LTP maintenance and effects on LTP expression. Furthermore, recent work casts strong doubt on whether ZIP has its effect by an action on PKM-zeta $[1,77,78]$. Importantly, recent work from the Sacktor laboratory demonstrates that the control peptide (scrambled ZIP) can also inhibit PKM-zeta and is only three times less effective than ZIP [79]. With such small differences in efficacy, it would take very precise administration of these peptides to produce a differential effect, but the concentrations used in in vivo experiments were highly imprecise (several orders of magnitude above the $K_{d}$ ) [1]. Thus, the differential effects on ZIP and scrambled ZIP are probably due to an effect on some target other than PKM-zeta. Finally, knockout of this enzyme did not affect late LTP, memory, or the effect of ZIP [80] (but see [81]). Thus, the evidence that PKM-zeta is the molecular basis of memory is not compelling.

\section{Conclusions}

The CaMKII/NMDAR complex is a promising candidate as the molecular basis of memory storage. Although there is increasing evidence that CaMKII and its binding partners form a molecular memory, additional work is required to prove this hypothesis. In particular, methods for studying the formation and persistence of the complex during actual LTP are needed. Furthermore, much additional work is required at the behavioral level to test the role of the CaMKII and the CaMKII/NMDAR complex in the persistence of memory. Most of the work on this complex has been done in the hippocampus Thus, it will be of particular importance to determine whether interference with the complex in the hippocampus can reverse a hippocampal-dependent spatial memory.

An important unresolved question is whether the importance of CaMKII/NMDAR complex in LTP maintenance, as demonstrated in Figure 1, will be specific for CA1 or more generally applicable to synapses. One perspective on this question comes from analysis of the PSDs, which are generally isolated from whole brains. Structural analysis shows that these PSDs are nearly tight-packed with CaMKII [82]. Given that these PSDs are representative of the whole brain, it seems likely that a critical role for CaMKII will be a widespread property. 
It has become increasingly clear that an important aspect of activity-dependent synaptic plasticity is the making and breaking of synaptic connections. Recent work shows that the ability of activity to stabilize synaptic connections is dependent on the CaMKII/NMDAR complex [83]. Thus, similar mechanisms may be involved in changes in synaptic strength and in stabilization of synaptic connections.

\section{Competing interests}

The authors declare that they have no competing interests.

\section{Authors' contributions}

$J L$ and MS contributed equally to writing this review. Both authors read and approved the final manuscript.

\section{Acknowledgements}

We thank Nikolai Otmakhov and Roger Colbran for comments on the manuscript. This work was supported by grants from the Ellison Foundation (J.L.), NIH Award Number R01DA027807 from the National Institute on Drug Abuse (J.L.), and University of Chile VID ENL-3/12 (M.S.).

\section{Author details}

'Department of Biology, Faculty of Sciences, University of Chile, Las Palmeras 3425, Santiago 7800024, Chile. ${ }^{2}$ Volen Center for Complex Systems, Biology Department, Brandeis University, 415 South Street, MS 008, Waltham, MA 02454-9110, USA.

Received: 6 December 2012 Accepted: 17 January 2013

Published: 14 February 2013

\section{References}

1. Lisman J, Yasuda R, Raghavachari S: Mechanisms of CaMKII action in longterm potentiation. Nat Rev Neurosci 2012, 13(3):169-182.

2. Faas $\mathrm{G}$, et al: Calmodulin as a direct detector of $\mathrm{Ca} 2+$ signals. Nat NeurosC 2011, 14(3):301-304

3. Benke TA, et al: Modulation of AMPA receptor unitary conductance by synaptic activity. Nature 1998, 393(6687):793-797.

4. Kristensen AS, et al: Mechanism of Ca2+/calmodulin-dependent kinase II regulation of AMPA receptor gating. Nat Neurosci 2011, 14(6):727-35.

5. Tomita $\mathrm{S}$, et al: Bidirectional synaptic plasticity regulated by phosphorylation of stargazin-like TARPs. Neuron 2005, 45(2):269-77.

6. Opazo $P$, et al: CaMKIl triggers the diffusional trapping of surface AMPARs through phosphorylation of stargazin. Neuron 2010, 67(2):239-252.

7. Omkumar RV, et al: Identification of a phosphorylation site for calcium/ calmodulindependent protein kinase II in the NR2B subunit of the N-methyl-D-aspartate receptor. J Biol Chem 1996, 271(49):31670-31678.

8. Strack S, Colbran RJ: Autophosphorylation-dependent targeting of calcium/ calmodulin-dependent protein kinase II by the NR2B subunit of the N-methyl- D-aspartate receptor. J Biol Chem 1998, 273(33):20689-20692.

9. Gardoni F, et al: Calcium/calmodulin-dependent protein kinase II is associated with NR2A/B subunits of NMDA receptor in postsynaptic densities. J Neurochem 1998, 71(4):1733-1741.

10. Leonard AS, et al: Calcium/calmodulin-dependent protein kinase II is associated with the N-methyl-D-aspartate receptor. Proc Natl Acad Sci USA 1999, 96(6):3239-3244.

11. Bayer KU, et al: Transition from reversible to persistent binding of CaMKII to postsynaptic sites and NR2B. J Neurosci 2006, 26(4):1164-1174

12. Appleby VJ, et al: LTP in hippocampal neurons is associated with a CaMKII-mediated increase in GluA1 surface expression. J Neurochem 2011, 116(4):530-543.

13. Otmakhov N, et al: Persistent accumulation of calcium/calmodulindependent protein kinase II in dendritic spines after induction of NMDA receptor-dependent chemical long-term potentiation. The Journal of neuroscience: the official journal of the Society for Neuroscience 2004, 24(42):9324-31.

14. Zhang YP, Holbro N, Oertner TG: Optical induction of plasticity at single synapses reveals input-specific accumulation of alphaCaMKII. Proc Natl Acad Sci USA 2008, 105(33):12039-44.
15. Lee SJ, Yasuda R: Spatiotemporal Regulation of Signaling in and out of Dendritic Spines: CaMKII and Ras. The open neuroscience journal 2009, 3:117-127.

16. Bayer KU, et al: Interaction with the NMDA receptor locks CaMKII in an active conformation. Nature 2001, 411(6839):801-5.

17. Feng B, Raghavachari S, Lisman J: Quantitative estimates of the cytoplasmic, PSD, and NMDAR-bound pools of CaMKII in dendritic spines. Brain Res 2011, 1419:46-52.

18. Tanaka J-I, et al: Protein synthesis and neurotrophin-dependent structural plasticity of single dendritic spines. Science 2008, 319(5870):1683-1687.

19. Fukunaga $K$, et al: Long-term potentiation is associated with an increased activity of $\mathrm{Ca} 2+/$ calmodulin-dependent protein kinase II. J Biol Chem 1993, 268(11):7863-7.

20. Barria A, Malinow R: NMDA receptor subunit composition controls synaptic plasticity by regulating binding to CaMKII. Neuron 2005, 48(2):289-301.

21. Strack S, McNeill RB, Colbran RJ: Mechanism and regulation of calcium/ calmodulin-dependent protein kinase II targeting to the NR2B subunit of the N-methyl-D-aspartate receptor. J Biol Chem 2000, 275(31):23798-806.

22. Halt $A$, et al: CaMKII binding to GluN2B is critical during memory consolidation. EMBO J 2012, 31(5):1203-1216.

23. Zhou Y, et al: Interactions between the NR2B receptor and CaMKI modulate synaptic plasticity and spatial learning. J Neurosci 2007, 27(50):13843-13853.

24. Pi HJ, et al: CaMKIl control of spine size and synaptic strength: role of phosphorylation states and nonenzymatic action. Proc Natl Acad Sci USA 2010, 107(32):14437-42.

25. Vest RS, et al: Dual mechanism of a natural CaMKII inhibitor. Mol Biol Cell 2007, 18(12):5024-33.

26. Chang BH, Mukherji S, Soderling TR: Characterization of a calmodulin kinase II inhibitor protein in brain. Proc Natl Acad Sci USA 1998, 95(18):10890-5

27. Sanhueza M, et al: Role of the CaMKII/NMDA receptor complex in the maintenance of synaptic strength. J Neurosci 2011, 31(25):9170-9178.

28. Dosemeci A, et al: The effect of CaMKII inhibitor tatCN21 on the re-distribution of CaMKIl and SynGAP in hippocampal neurons under excitatory conditions. SFN Program\#/Poster\#: 43.15/C53. In Society for Neuroscience. New Orleans; 2012.

29. Otmakhov N, Griffith LC, Lisman JE: Postsynaptic inhibitors of calcium/ calmodulin-dependent protein kinase type II block induction but not maintenance of pairing-induced long-term potentiation. J Neurosci 1997, 17(14):5357-5365.

30. Chen HX, et al: Is persistent activity of calcium/calmodulin-dependent kinase required for the maintenance of LTP? J Neurophysiol 2001 85(4):1368-1376.

31. Mullasseril $P$, et al: A structural mechanism for maintaining the 'on-state' of the CaMKII memory switch in the post-synaptic density. J Neurochem 2007, 103(1):357-64.

32. Gruart A, Munoz MD, Delgado-Garcia JM: Involvement of the CA3-CA1 synapse in the acquisition of associative learning in behaving mice. The Journal of neuroscience: the official journal of the Society for Neuroscience 2006, 26(4):1077-87.

33. Whitlock JR, et al: Learning induces long-term potentiation in the hippocampus. Science 2006, 313(5790):1093-7.

34. Liao D, Jones A, Malinow R: Direct measurement of quantal changes underlying long-term potentiation in CA1 hippocampus. Neuron 1992, 9(6):1089-1097.

35. Debanne D, Gahwiler BH, Thompson SM: Heterogeneity of synaptic plasticity at unitary CA3-CA1 and CA3-CA3 connections in rat hippocampal slice cultures. The Journal of neuroscience: the official journal of the Society for Neuroscience 1999, 19(24):10664-71.

36. Gouet C, et al: On the Mechanism of Synaptic Depression Induced by CaMKIIN, an Endogenous Inhibitor of CaMKII. PLoS One 2012, 7(11):e49293.

37. Petralia RS, et al: Ontogeny of postsynaptic density proteins at glutamatergic synapses. Mol Cell Neurosci 2005, 29(3):436-52.

38. Swulius MT, et al: Structure and composition of the postsynaptic density during development. J Comp Neurol 2010, 518(20):4243-60.

39. Bailey $\mathrm{CH}$, Kandel ER: Synaptic remodeling, synaptic growth and the storage of long-term memory in Aplysia. Prog Brain Res 2008, 169:179-198.

40. Bourne JN, Harris KM: Coordination of size and number of excitatory and inhibitory synapses results in a balanced structural plasticity along 
mature hippocampal CA1 dendrites during LTP. Hippocampus 2011, 21(4):354-73.

41. Frey U, Morris RG: Synaptic tagging and long-term potentiation. Nature 1997, 385(6616):533-536.

42. Garic A, et al: CaMKIl activation is a novel effector of alcohol's neurotoxicity in neural crest stem/progenitor cells. J Neurochem 2011, 118(4):646-57.

43. Redondo RL, et al: Synaptic tagging and capture: differential role of distinct calcium/calmodulin kinases in protein synthesis-dependent long-term potentiation. The Journal of neuroscience: the official journal of the Society for Neuroscience 2010, 30(14):4981-9.

44. Bozdagi O, et al: Increasing numbers of synaptic puncta during latephase LTP: N-cadherin is synthesized, recruited to synaptic sites, and required for potentiation. Neuron 2000, 28(1):245-59.

45. Bozdagi O, et al: Persistence of coordinated long-term potentiation and dendritic spine enlargement at mature hippocampal CA1 synapses requires $\mathrm{N}$-cadherin. The Journal of neuroscience: the official journal of the Society for Neuroscience 2010, 30(30):9984-9.

46. Tang L, Hung CP, Schuman EM: A role for the cadherin family of cell adhesion molecules in hippocampal long-term potentiation. Neuron 1998, 20(6):1165-75.

47. Mendez $\mathrm{P}$, et al: N-cadherin mediates plasticity-induced long-term spine stabilization. J Cell Biol 2010, 189(3):589-600.

48. Walikonis RS, et al: Densin-180 forms a ternary complex with the (alpha)subunit of $\mathrm{Ca} 2+/$ calmodulin-dependent protein kinase II and (alpha)actinin. The Journal of neuroscience: the official journal of the Society for Neuroscience 2001, 21(2):423-33.

49. Robison AJ, et al: Multivalent Interactions of Calcium/Calmodulin-dependent Protein Kinase II with the Postsynaptic Density Proteins NR2B, Densin-180, and a-Actinin-2. J Biol Chem 2005, 280(42):35329-35336.

50. Izawa I, et al: Densin-180 interacts with delta-catenin/neural plakophilinrelated armadillo repeat protein at synapses. J Biol Chem 2002, 277(7):5345-50.

51. Brigidi GS, Bamji SX: Cadherin-catenin adhesion complexes at the synapse. Curr Opin Neurobiol 2011, 21(2):208-14.

52. Lisman JE, Harris KM: Quantal analysis and synaptic anatomy-integrating two views of hippocampal plasticity. Trends Neurosci 1993, 16(4):141-7.

53. Lisman J: The pre/post LTP debate. Neuron 2009, 63(3):281-284.

54. Lisman J, Raghavachari S: A unified model of the presynaptic and postsynaptic changes during LTP at CA1 synapses. Science's signal transduction knowledge environment 2006, 2006(356):re11-re11.

55. Silverman $\mathrm{JB}$, et al: Synaptic anchorage of AMPA receptors by cadherins through neural plakophilin-related arm protein-AMPA receptor-binding protein complexes. J Neurosci 2007, 27(32):8505-8516.

56. DeSouza S, et al: Differential Palmitoylation Directs the AMPA ReceptorBinding Protein ABP to Spines or to Intracellular Clusters. J Neurosci 2002, 22(9):3493-3503.

57. Ochiishi T, et al: Regulation of AMPA receptor trafficking by $\delta$-catenin. Mol Cell Neurosci 2008, 39(4):499-507.

58. Israely l, et al: Deletion of the neuron-specific protein delta-catenin leads to severe cognitive and synaptic dysfunction. Current biology: CB 2004, 14(18):1657-63.

59. Saglietti $\mathrm{L}$, et al: Extracellular interactions between GluR2 and N-cadherin in spine regulation. Neuron 2007, 54(3):461-77.

60. Zhou Z, et al: GluA2 (GluR2) regulates metabotropic glutamate receptordependent long-term depression through $\mathrm{N}$-cadherin-dependent and cofilin-mediated actin reorganization. The Journal of neuroscience: the official journal of the Society for Neuroscience 2011, 31(3):819-33.

61. Vitureira $\mathrm{N}$, et al: Differential control of presynaptic efficacy by postsynaptic $\mathrm{N}$-cadherin and [beta]-catenin. Nat Neurosci 2012, 15(1):81-89.

62. Lu FM, Hawkins RD: Presynaptic and postsynaptic $\mathrm{Ca}(2+)$ and CamKII contribute to long-term potentiation at synapses between individual CA3 neurons. Proc Natl Acad Sci USA 2006, 103(11):4264-9.

63. Shakiryanova D, et al: Differential control of presynaptic CaMKIl activation and translocation to active zones. The Journal of neuroscience: the official journal of the Society for Neuroscience 2011, 31(25):9093-100.

64. Jalan-Sakrikar N, et al: Substrate-selective and calcium-independent activation of CaMKII by alpha-actinin. J Biol Chem 2012, 287(19):15275-83.

65. Strack S, et al: Association of calcium/calmodulin-dependent kinase II with developmentally regulated splice variants of the postsynaptic density protein densin-180. J Biol Chem 2000, 275(33):25061-4.
66. Carlisle HJ, et al: Deletion of densin-180 results in abnormal behaviors associated with mental illness and reduces mGluR5 and DISC1 in the postsynaptic density fraction. The Journal of neuroscience: the official journal of the Society for Neuroscience 2011, 31(45):16194-207.

67. Loweth $\mathrm{JA}$, et al: Persistent Reversal of Enhanced Amphetamine Intake by Transient CaMKII Inhibition. The Journal of neuroscience: the official journal of the Society for Neuroscience 2013, 33(4):1411-6.

68. Shen K, Meyer T: Dynamic control of CaMKII translocation and localization in hippocampal neurons by NMDA receptor stimulation. Science 1999, 284(5411):162-6.

69. Yamagata $Y$, et al: Kinase-dead knock-in mouse reveals an essential role of kinase activity of $\mathrm{Ca} 2+/$ calmodulin-dependent protein kinase llalpha in dendritic spine enlargement, long-term potentiation, and learning. J Neurosci 2009, 29(23):7607-7618.

70. O'Leary $\mathrm{H}$, et al: Nucleotides and phosphorylation bi-directionally modulate Ca2+/calmodulin-dependent protein kinase II (CaMKII) binding to the N-methyl-D-aspartate (NMDA) receptor subunit GluN2B. J Biol Chem 2011, 286(36):31272-81.

71. Wang $\mathrm{H}$, et al: Inducible protein knockout reveals temporal requirement of CaMKII reactivation for memory consolidation in the brain. Proc Natl Acad Sci USA 2003, 100(7):4287-92.

72. Jiang $\mathrm{X}$, et al: Modulation of CaV2.1 channels by Ca2+/calmodulindependent protein kinase II bound to the C-terminal domain. Proc Natl Acad Sci USA 2008, 105(1):341-346.

73. Li Y, et al: Phosphorylated CaMKII post-synaptic binding to NR2B subunits in the anterior cingulate cortex mediates visceral pain in visceral hypersensitive rats. J Neurochem 2012, 121(4):662-671.

74. Sacktor T: Memory maintenance by PKMzeta - an evolutionary perspective. Mol Brain 2012, 5(1):31-31.

75. Serrano P, Yao Y, Sacktor T: Persistent phosphorylation by protein kinase Mzeta maintains late-phase long-term potentiation. J Neurosci 2005, 25(8):1979-1984.

76. Pastalkova $\mathrm{E}$, et al: Storage of spatial information by the maintenance mechanism of LTP. Science 2006, 313(5790):1141-4.

77. Wu-Zhang A, et al: Cellular pharmacology of protein kinase $M \zeta$ (PKMZ) contrasts with its in vitro profile: implications for PKM as a mediator of memory. J Biol Chem 2012, 287(16):12879-12885

78. Lisman J: Memory erasure by very high concentrations of ZIP may not be due to PKM-zeta. Hippocampus 2012, 22(3):648-649.

79. Yao Y, et al: Matching biochemical and functional efficacies confirm ZIP as a potent competitive inhibitor of $\mathrm{PKM} \zeta$ in neurons. Neuropharmacology 2013, 64:37-44

80. Volk $L$, et al: PKM-zeta is not required for hippocampal synaptic plasticity, learning and memory. Nature 2013, 493(7432):420-3.

81. Tsokas P, et al Conditional knockout of the PKC/PKM gene in the adult mouse hippocampus prevents L-LTP. in 2012 Society for Neuroscience Meeting: New Orleans. SfN: LA; 2012.

82. Petersen JD, et al: Distribution of Postsynaptic Density (PSD)-95 and $\mathrm{Ca} 2+/$ Calmodulin-Dependent Protein Kinase II at the PSD. J Neurosci 2003, 23(35):11270-11278.

83. Hamilton AM, et al: Activity-dependent growth of new dendritic spines is regulated by the proteasome. Neuron 2012, 74(6):1023-30.

doi:10.1186/1756-6606-6-10

Cite this article as: Sanhueza and Lisman: The CaMKII/NMDAR complex as a molecular memory. Molecular Brain 2013 6:10. 